\title{
Sympathetic Modulation of the Host Defense Response to Infectious Challenge during Recovery from Hemorrhage
}

\author{
Annie M. Whitaker Jesse Sulzer Edith Walker Keisa Mathis Patricia E. Molina \\ Department of Physiology, Louisiana State University Health Science Center, and Alcohol and Drug Abuse Center \\ of Excellence, New Orleans, La., USA
}

\section{Key Words}

Cecal ligation/puncture $\cdot$ Hemorrhage $\cdot$ Inflammation .

Noradrenergic system $\cdot$ Sympathectomy $\cdot$ Trauma

\begin{abstract}
Background: Trauma/hemorrhage (TxHem) is associated with an immediate pro-inflammatory response that, if exaggerated or prolonged, is thought to contribute to the subsequent immunosuppression that characterizes the period after injury. Previously we have demonstrated that chemical sympathectomy (SNSx) accentuates this immediate pro-inflammatory response to TxHem. These findings suggest that the noradrenergic system plays a critical role in limiting the magnitude of the inflammatory response during TxHem and preserving the integrity of the host defense response to a subsequent infectious challenge during the period after TxHem. Objective: To examine the contribution of tissue norepinephrine to the host defense response to an infectious challenge during recovery from TxHem. Methods: Male Sprague-Dawley rats underwent SNSx (6-hydroxydopamine, i.p. daily for 3 days) prior to vascular catheter implantation. Conscious, unrestrained rats were subjected to traumatic injury (muscle crush) prior to a fixed-pressure hemorrhage $(40 \mathrm{~mm} \mathrm{Hg}$ for $60 \mathrm{~min}$ ) and fluid resuscitation followed $24 \mathrm{~h}$ later by cecal ligation and puncture (CLP). $\boldsymbol{R e}$ sults: SNSx impaired the hemodynamic and thermoregu-
\end{abstract}

latory response to hemorrhage as indicated by decreased basal blood pressure, impaired blood pressure recovery during fluid resuscitation, and greater hypothermia after CLP. Furthermore, SNSx accentuated the TNF- $\alpha$, IL-1, IL- 6 , and IL10 response to TxHem + infection in plasma $6 \mathrm{~h}$ after CLP and in peritoneal lavage fluid $24 \mathrm{~h}$ after CLP. Conclusion: These results indicate that the integrity of the noradrenergic system is necessary for adequate hemodynamic, thermoregulatory, and inflammatory responses to infection during the period following TxHem.

Copyright $\odot 2010$ S. Karger AG, Basel

\section{Introduction}

Sympathetic nervous system (SNS) activation plays a role in the response to injury and blood loss by contributing to the restoration of hemodynamic stability and maintenance of adequate tissue perfusion [1]. In addition, this descending pathway has been shown to regulate immune cell function [2-4]. Anatomic evidence demonstrates significant innervation from sympathetic postganglionic neurons to immune tissues such as the thymus, spleen, and lymph nodes where they interact with immune cells via release of norepinephrine in synaptic and non-synaptic pathways $[5,6]$. 
Results from in vitro studies suggest that the effects of adrenergic stimulation of immune cells are receptor and cell specific. While under most conditions $\alpha$-adrenergic receptor activation augments pro-inflammatory cytokine production $[2,7,8]$, activation of $\beta_{2}$-adrenergic receptors results in immunosuppression by decreasing the release of pro-inflammatory cytokines $[2,9]$. These studies provide strong evidence of adrenergic regulation of immune cell function; however, studies examining adrenergic modulation of the host response in vivo are limited. Studies have demonstrated that circulating catecholamines regulate the immune response to lipopolysaccharides (LPS) [10]. In addition, sympathectomy (SNSx) has been shown to increase the number of peritoneal macrophages following an intraperitoneal infection with Listeria monocytogenes [11]. These studies provide in vivo evidence of sympathetic modulation of the immune response to LPS and infection; however, the contribution of noradrenergic innervation on the immune response to sepsis, particularly after hemorrhagic shock, remains unknown. Sepsis during recovery from traumatic injury and blood loss remains a significant cause of late mortality [12]. Given that there is an increased susceptibility to infection following hemorrhagic shock [13], it is critical to understand the contribution of noradrenergic innervation to the host defense response to infection during the recovery period.

Previous studies from our laboratory have shown that depletion of tissue norepinephrine by chemical SNSx significantly enhances the hemorrhage-induced rise in lung TNF- $\alpha$ content [14]. These findings suggest that SNS activation, through localized tissue norepinephrine release, regulates the magnitude of the early pro-inflammatory cytokine response to hemorrhage. Therefore, we hypothesized that the integrity of the noradrenergic system is essential in limiting the magnitude and duration of the early pro-inflammatory response and in turn preserving the integrity of host defense mechanisms during recovery from injury. The aim of the present study was to examine the contribution of tissue norepinephrine content on the inflammatory response to sepsis following trauma/hemorrhage (TxHem). Our findings indicate that the noradrenergic system plays a critical role in regulating the magnitude of the pro-inflammatory response to infection. Thus, we conclude that noradrenergic activation during traumatic injury and hemorrhage, in addition to playing a central role in the counterregulatory response to blood loss, also contributes to regulation of the inflammatory response to infection during the early recovery period.

\section{Materials and Methods}

\section{Animal Preparation}

All experiments and animal procedures were approved by the Institutional Animal Care and Use Committee of the Louisiana State University Health Science Center and were in accordance with the National Institutes of Health guidelines. Specific pathogen-free adult male Sprague-Dawley rats (Charles River, Raleigh, N.C., USA) weighing 275-300 g were housed in the Division of Animal Care at the Louisiana State University Health Science Center in a controlled environment for 1 week prior to experimental procedures to allow the animals to acclimate to their surroundings. Animals were exposed to a 12-hour light/12-hour dark cycle and fed standard rat diet (Purina Rat Chow; Ralston Purina, St. Louis, Mo., USA).

Noradrenergic Depletion

Chemical SNSx was achieved by administration of 6-hydroxydopamine (6-OHDA; Sigma, St. Louis, Mo., USA). 6-OHDA is a neurotoxin that destroys noradrenergic nerve terminals by induction of oxidative stress. Several small doses were administered to ensure specificity for noradrenergic neurons [15]. Intraperitoneal injections of $50 \mathrm{mg} / \mathrm{kg} 6$-OHDA (dissolved in $0.2 \%$ ascorbic acid) were administered for 3 consecutive days prior to vascular catheter placement ( 9 days prior to the experiment). A fourth injection of $100 \mathrm{mg} / \mathrm{kg}$ was administered 3 days prior to the experiment. Time-matched controls received injections of equal volumes of the vehicle $(0.2 \%$ ascorbic acid, $\sim 300 \mu \mathrm{l})$. We have previously demonstrated that this protocol results in $80-90 \%$ depletion of tissue norepinephrine [14].

Surgical Preparation

Rats were anesthetized with an intramuscular injection of ketamine/xylazine (90 and $9 \mathrm{mg} / \mathrm{kg}$, respectively) and implanted with sterile vascular catheters (left carotid artery advanced to the aortic arch and right jugular vein; PE50) using aseptic surgical techniques, as previously described by our laboratory [16]. The catheters were flushed with normal saline and routed subcutaneously to the nape of the neck where they were exteriorized through a small incision and secured with tape. After surgery, the animals were placed in individual cages, allowed to recover from anesthesia, and given food and water ad libitum.

\section{TxHem Protocol}

Experiments were started at approximately 7 a.m. 4 days after surgery and 3 days after the fourth injection of 6-OHDA. Animals were conscious and unrestrained throughout the duration of the hemorrhage. Animals were randomized into four groups: vehicle/ sham, vehicle/TxHem, SNSx/sham, and SNSx/TxHem. Sham animals were surgically implanted with vascular catheters but were not subjected to TxHem. A model of trauma and fixed-pressure hemorrhage, previously described by our laboratory, was used in these studies [17]. Animals were anesthetized with Brevital (25 mg/ $\mathrm{kg}$, i.v.) and a full-thickness gastrocnemius muscle crush was performed for a $3 \mathrm{~min}$ duration prior to hemorrhage. Once the animals were fully recovered from Brevital ( $\sim 15 \mathrm{~min})$, they were subjected to a fixed-pressure hemorrhage. The carotid catheter was exteriorized and connected to a pressure transducer (MacLab, Colorado Springs, Colo., USA) for continuous blood pressure recording. Animals were bled by withdrawing blood from the carotid 
catheter into a heparinized syringe to achieve a mean arterial blood pressure (MABP) of $\sim 40 \mathrm{~mm} \mathrm{Hg}$. Additional blood was removed to maintain MABP between 40 and $55 \mathrm{~mm} \mathrm{Hg}$ for $60 \mathrm{~min}$. At the completion of hemorrhage, animals were resuscitated with warmed Ringer's lactate. A bolus of $40 \%$ of the total blood volume removed was administered intravenously followed by a constant infusion replacing two times the total blood volume removed over $60 \mathrm{~min}$. Blood samples were obtained at baseline, after TxHem, and at completion of fluid resuscitation for determination of circulating corticosterone and catecholamines. At the end of the fluid resuscitation period, catheters were flushed with $1 \mathrm{ml}$ of normal saline, disconnected from the pressure transducers, and secured at the nape of the neck. Animals were returned to their individual cages and provided food and water ad libitum overnight prior to undergoing cecal ligation and puncture (CLP) the following morning.

\section{CLP Protocol}

To examine the impact of chemical SNSx on the host response to an infectious challenge during the recovery phase following TxHem, animals were subjected to CLP as a model of polymicrobial peritoneal infection $24 \mathrm{~h}$ following TxHem and fluid resuscitation [18]. Animals were anesthetized with an intramuscular injection of ketamine/xylazine ( 90 and $9 \mathrm{mg} / \mathrm{kg}$, respectively). A midline abdominal incision was performed to expose the cecum. The cecum was ligated below the ileocecal valve without obstructing the bowel and punctured twice with a 20 -gauge needle. Fecal material was gently expressed and $1.5 \mathrm{ml}$ of normal saline were injected into the abdominal cavity prior to closure of the incision in two planes. After surgery, animals were returned to individual cages to recover and provided food and water ad libitum. Six and $24 \mathrm{~h}$ after CLP, MABP and rectal temperature were recorded and a blood sample collected for determination of plasma cytokines. Twenty-four hours after CLP, following the final MABP and temperature measurements, animals were anesthetized and peritoneal lavage was performed. Briefly, $15 \mathrm{ml}$ of heparinized PBS $(2,000 \mathrm{U} / \mathrm{l})$ were instilled into the peritoneal cavity using a sterile technique and the abdomen gently massaged prior to fluid recovery. Average volume recovered was $\sim 10 \mathrm{ml}$. Lavage fluid was centrifuged at $650 \mathrm{~g}$ for $10 \mathrm{~min}$ and the supernatant stored at $-80^{\circ} \mathrm{C}$ until cytokine analysis. The lung and spleen were immediately excised, washed in normal saline, freeze clamped with liquid nitrogen, and stored at $-80^{\circ} \mathrm{C}$ until analyzed.

\section{Analytical Procedures}

Arterial blood samples were collected in chilled, heparinized syringes. Blood was placed in tubes containing either aprotinin (Sigma, St. Louis, Mo., USA) at $10 \mu \mathrm{l} / \mathrm{ml}$ of blood or catecholamine preservative (9\% EDTA, $6 \%$ glutathione, and $\mathrm{dH}_{2} \mathrm{O}$ at a $\mathrm{pH}$ value of $6.0-7.4)$ at $20 \mu \mathrm{l} / \mathrm{ml}$ of blood. Blood was centrifuged for $10 \mathrm{~min}$ at $10,000 \mathrm{rpm}$ for plasma separation. Plasma was collected and stored at $-80^{\circ} \mathrm{C}$ for analysis of catecholamines, hormones, and cytokines. Plasma catecholamines (epinephrine and norepinephrine) were determined by high-performance liquid chromatography as previously described by our laboratory [14]. Corticosterone levels were measured as an index of activation of the hypothalamo-pituitary-adrenal axis using a rat-specific radioimmunoassay (Diagnostic Products, Los Angeles, Calif., USA). Plasma, tissue and peritoneal lavage cytokines were measured using a ratspecific ELISA (Biosource International, Carlsbad, Calif., USA) or multiplex cytokine panel (Invitrogen, Carlsbad, Calif., USA).

\section{Tissue Preparation}

Tissues were prepared for cytokine assays as described by our laboratory [17]. Briefly, frozen tissue samples were weighed and placed in homogenization buffer $\left(4^{\circ} \mathrm{C}\right)$ at a ratio of $100 \mathrm{mg}$ tissue/ $\mathrm{ml}$ of buffer. Samples were homogenized (Polytron; Brinkman, Westbury, N.Y., USA), subjected to one freeze-thaw cycle, sonicated for $10 \mathrm{~min}$ and incubated at $4^{\circ} \mathrm{C}$ for $1 \mathrm{~h}$. The final homogenate was centrifuged at $120,000 \mathrm{~g}$ (Beckman Ultracentrifuge, model L855) for $25 \mathrm{~min}$ at $4^{\circ} \mathrm{C}$. Tissue cytokine content was measured in the supernatant. Tissue protein content was determined by the procedure of Lowry et al. [19]. Tissue cytokine content is expressed per milligram of tissue protein.

\section{Statistical Analysis}

All data are expressed as means \pm SEM with the number of animals per group indicated ( $n=5-10$ per group). Statistical analyses of MABP, tissue, peritoneal lavage, and plasma cytokines, plasma catecholamines and corticosterone, and rectal temperature were determined by two-way analysis of variance with or without repeated measures. Statistical significance was set at $\mathrm{p}<0.05$.

\section{Results}

\section{Impact of SNSx on Response to TxHem}

Baseline MABP averaged at $126 \pm 3 \mathrm{~mm} \mathrm{Hg}$ in control animals (fig. 1). Chemical SNSx produced a significant decrease in basal MABP to an average of $107 \pm 4 \mathrm{~mm} \mathrm{Hg}$. SNSx did not change the rate of drop in pressure in response to blood loss nor the percent of total blood volume required to achieve an average fixed pressure of $55 \mathrm{~mm} \mathrm{Hg}$. Blood pressure recovery during fluid resuscitation was significantly impaired in SNSx animals. MABP was 10\% lower in SNSx animals compared to vehicle-treated animals during the fluid resuscitation period. Basal rectal temperature was not different between the groups and showed a similar decrease of 5 and $6 \%$ in vehicle-treated and SNSx groups, respectively, at the end of TxHem (fig. 2).

\section{Impact of SNSx on Neuroendocrine Response to \\ TxHem}

SNSx did not alter basal levels of circulating norepinephrine (fig. 3). TxHem produced a 2 -fold increase in circulating norepinephrine in vehicle-treated animals which was significantly blunted by SNSx. This response was consistent throughout the fluid resuscitation period. Epinephrine and corticosterone levels were increased by TxHem (3- and 4- to 5-fold, respectively) and were unaltered by prior SNSx.

\section{Impact of SNSx on Response to CLP}

CLP produced a significant decrease in MABP in both vehicle-treated and SNSx animals $6 \mathrm{~h}$ after CLP. Twenty- 


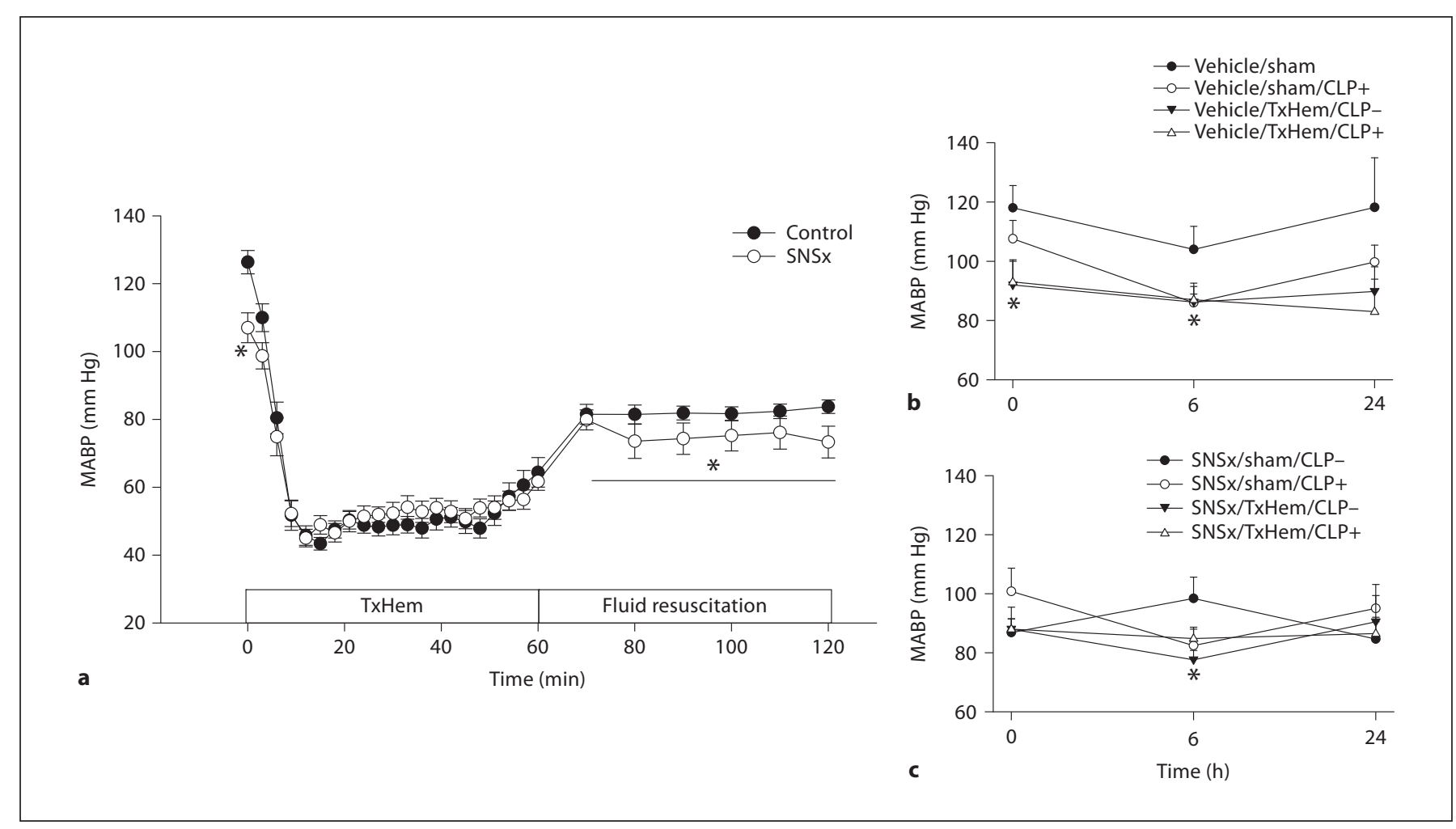

Fig. 1. MABP as a function of time during hemorrhage and fluid resuscitation in chemical SNSx and timematched control animals (a) and following CLP in vehicle (b) and SNSx (c) animals. Means \pm SEM, $\mathrm{n}=21-30$ / group. ${ }^{*} \mathrm{p}<0.05$ vs. time-matched controls.

four hours after CLP, MABP in all experimental groups had returned to values obtained after TxHem. Rectal temperature was not different between experimental groups prior to CLP (fig. 2), but showed a transient decrease $6 \mathrm{~h}$ after CLP in vehicle-treated animals. The decrease in temperature was accentuated in SNSx animals. Twenty-four hours after CLP, rectal temperature in vehicle-treated animals had returned to basal values while SNSx animals remained hypothermic.

\section{Impact of SNSx on Inflammatory Response to CLP}

Plasma levels of TNF- $\alpha$, IL-1 $\beta$, IL-6, and IL-10 were measured $6 \mathrm{~h}$ after CLP (fig. 4). SNSx did not alter unstimulated cytokine levels. Furthermore, TxHem alone did not affect cytokine levels $6 \mathrm{~h}$ after CLP in both vehicle and SNSx animals. CLP alone produced a significant increase in all four cytokines in vehicle-treated sham animals $6 \mathrm{~h}$ after CLP. In contrast, vehicle-treated TxHem animals did not show a significant CLP-induced increase in any of the cytokines measured. SNSx animals showed a significant increase in TNF- $\alpha$ and IL- 6 levels $6 \mathrm{~h}$ after
CLP. The response to CLP was further accentuated in animals that had undergone TxHem prior to CLP. Thus, SNSx animals subjected to TxHem prior to CLP had a significantly accentuated increase in all cytokines measured compared to their vehicle-treated treatmentmatched controls.

Tissue cytokine content of lung and spleen was measured $24 \mathrm{~h}$ after CLP. In the lung, SNSx did not alter unstimulated cytokine content (fig. 5). TxHem produced a significant increase in lung TNF- $\alpha$, IL- $1 \alpha$, and IL- 6 in vehicle-treated animals and a significant increase in lung TNF- $\alpha$ and IL- $1 \alpha$ of SNSx animals. CLP produced a significant increase in IL- $1 \alpha$ and IL- 6 in the lung of vehicletreated animals, and in lung IL- $1 \alpha$ of SNSx animals. The CLP-induced increase in IL- $1 \alpha$ was significantly enhanced compared to treatment-matched vehicle-treated animals. TxHem prior to CLP resulted in a significant increase in lung TNF- $\alpha$ and IL-1 $\alpha$ in vehicle-treated animals and a significant increase in all cy tokines measured in SNSx animals. The TNF- $\alpha$ and IL- $1 \alpha$ response to TxHem in combination with CLP in SNSx animals was 
significantly enhanced compared to treatment-matched vehicle-treated animals.

In the spleen, SNSx did not alter unstimulated cytokine content (fig. 6). TxHem produced a significant increase in TNF- $\alpha$ and IL- 6 in vehicle-treated animals and TNF- $\alpha$ in SNSx animals. CLP produced a significant increase in all of the cytokines measured in vehicle-treated animals and in TNF- $\alpha$, IL-1 $\alpha$, and IL-10 in SNSx animals. TxHem prior to CLP produced a significant increase in IL-1 $\alpha$ and IL-10 in vehicle-treated animals and in all cytokines measured in SNSx animals. However, SNSx did not modify the cytokine response of the spleen compared to vehicle-treated injury-matched controls.

Local-compartment immune modulation was assessed by measuring the cytokine content of peritoneal lavage samples collected $24 \mathrm{~h}$ after CLP (fig. 7). In both vehicle-treated and SNSx groups, CLP alone increased IL- 6 and IL-10 levels, while the early markers of inflammation, TNF- $\alpha$ and IL-1 $\alpha$ were not significantly different. SNSx in the TxHem group enhanced the cytokine response to CLP in the peritoneal cavity. Both pro-inflammatory (TNF- $\alpha$ and IL-6) and anti-inflammatory (IL-10) cytokine levels were significantly increased compared to vehicle-treated, treatment-matched controls.

\section{Discussion}

We examined the contribution of tissue norepinephrine content on the inflammatory response to sepsis following TxHem. In agreement with previous findings, the results from this study demonstrate a significant role of tissue norepinephrine content in the initial response to TxHem as evidenced by a decrease in basal MABP and impaired blood pressure recovery following hemorrhagic shock in SNSx animals [14]. In response to a 'second-hit' infectious challenge, sympathectomized animals demonstrated a significant dysregulation of the inflammatory response characterized by accentuated CLP-induced rises in the pro-inflammatory cytokines TNF- $\alpha$, IL-6, and IL-1 $\beta$, as well as the anti-inflammatory IL-10 in plasma $6 \mathrm{~h}$ after infection. Peritoneal lavage TNF- $\alpha$, IL-6, and IL-10 content $24 \mathrm{~h}$ after CLP was also significantly accentuated by prior SNSx. This was accompanied by enhanced TNF- $\alpha$ and IL-1 content in the lung compared to vehicle-treated animals. Together these findings suggest that tissue norepinephrine, a key component of the SNS response to trauma and hemorrhage, continues to contribute to the regulation of the inflammatory response during the early recovery period.

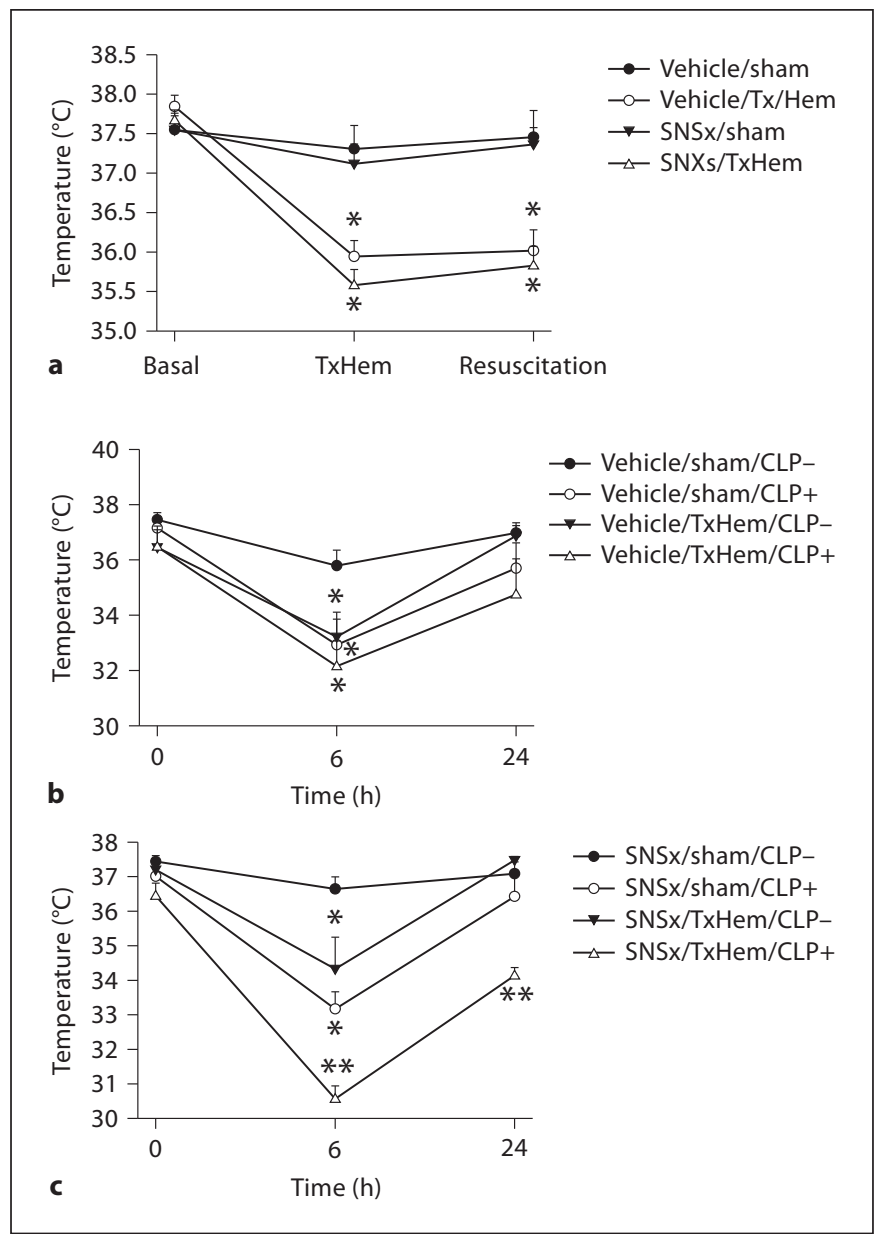

Fig. 2. Rectal temperature in response to TxHem (a) and following CLP in chemical SNSx (c) and time-matched control (b) animals. Means \pm SEM, $n=7-8$ /group. ${ }^{*} \mathrm{p}<0.05$ vs. baseline or time $=0$, ${ }^{* *} \mathrm{p}<0.05$ vs. time-matched control.

The noradrenergic system has been shown to produce receptor-specific modulation of immune function [20]. In vitro studies show that $\alpha$-adrenergic stimulation of macrophages leads to increased TNF- $\alpha$ message expression, while $\beta$-adrenergic stimulation inhibits the production of TNF- $\alpha$, IL-1 $\beta$, and IL-6 [8]. Among the mechanisms that have been identified to be involved in noradrenergic inhibition of inflammatory responses is modulation of IкB/NFKB signaling. Activation of $\beta_{2}$-adrenergic receptors has been shown to decrease NFKB-mediated transcription of pro-inflammatory cytokines [2123]. Moreover, $\beta$-adrenergic agonists abolish LPS-induced pro-inflammatory mRNA expression and gene transcription in monocytic cells through a cAMP-mediated mechanism $[21,22]$ involving signaling through

Neuroimmunomodulation 2010;17:349-358 


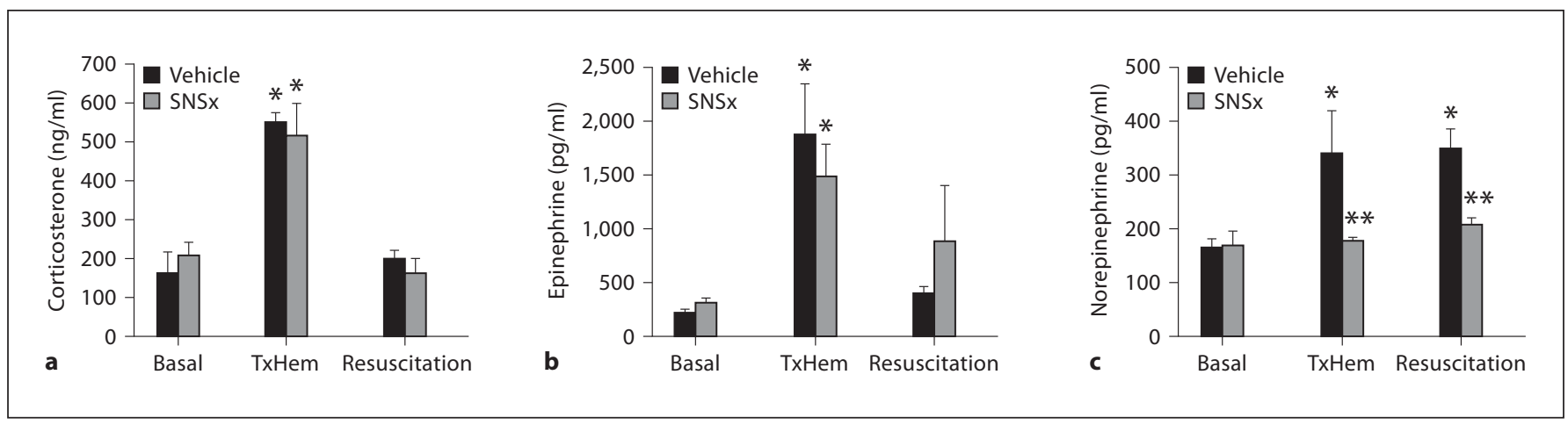

Fig. 3. Plasma corticosterone (a), epinephrine (b), and norepinephrine (c) at baseline, at completion of TxHem, and at completion of fluid resuscitation in control and SNSx animals. Means \pm SEM, $n=7-9 /$ group. ${ }^{*} \mathrm{p}<0.05$ vs. baseline, ${ }^{* *} \mathrm{p}<0.05$ vs. time-matched controls.

Fig. 4. Plasma levels of TNF- $\alpha$ (a), IL-1 $\beta$ (b), IL-6 (c), and IL-10 (d) obtained from sham \pm CLP and TxHem \pm CLP in control and SNSx (6-OHDA i.p.) groups. Plasma was obtained $6 \mathrm{~h}$ after CLP. Means \pm SEM, $\mathrm{n}=8-10 /$ group. ${ }^{*} \mathrm{p}<0.05$ vs. timematched sham/CLP-, ${ }^{* *} \mathrm{p}<0.05$ vs. timematched treatment control.

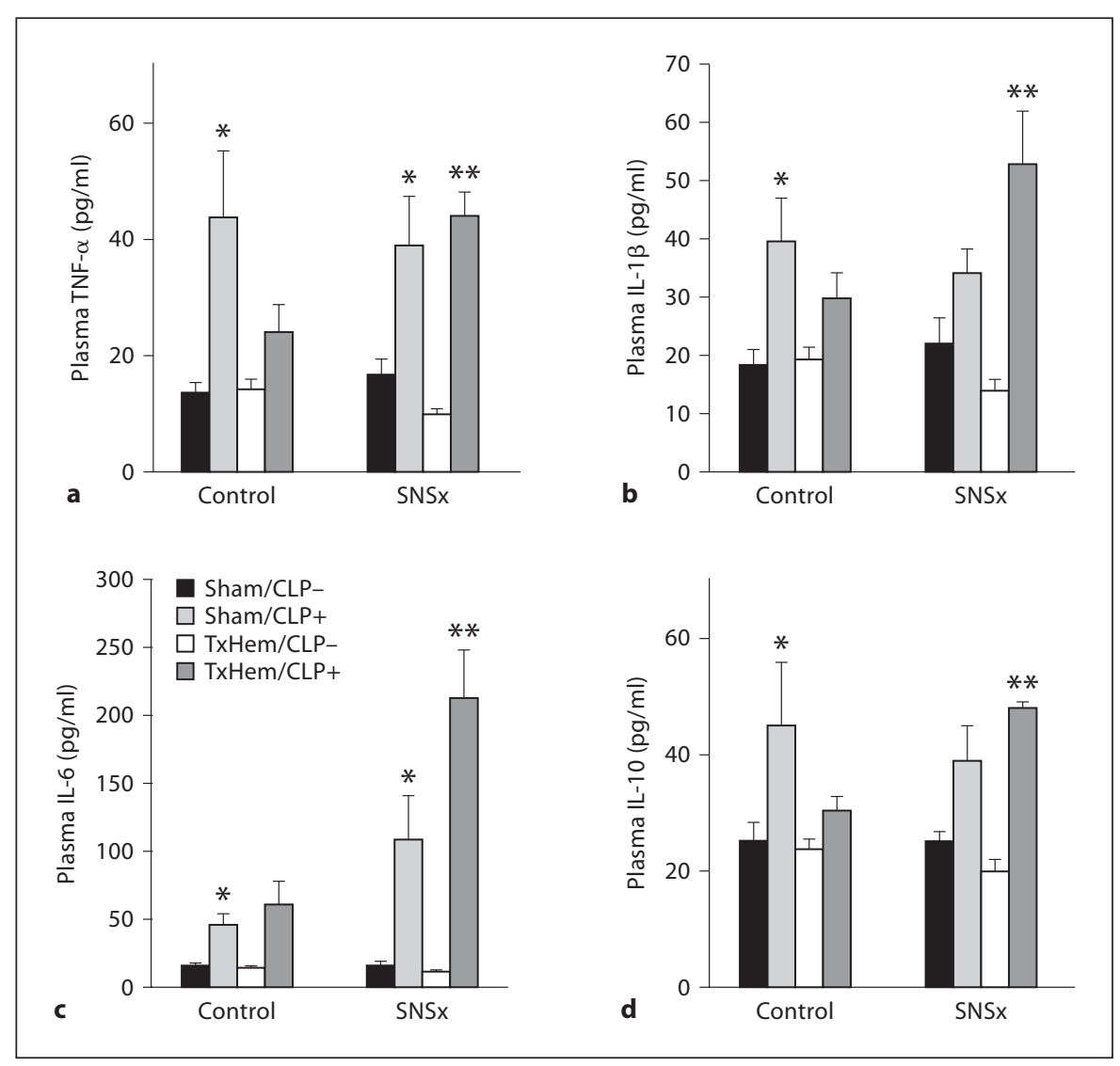

protein kinase A. Protein kinase A inhibits NFкB-mediated transcription by phosphorylating cAMP response element-binding protein (CREB) which competes with $\mathrm{NF \kappa B}$ proteins for binding to the transcriptional co-activator CREB-binding protein resulting in decreased acti- vation of $\mathrm{NF \kappa B}$ [24]. Others have demonstrated that the $\beta$-adrenergic agonist isoproterenol increases cytosolic ІкB- $\alpha$ in LPS-stimulated monocytes thereby preventing NFKB translocation and transcription of pro-inflammatory cytokines [21]. 
Fig. 5. TNF- $\alpha$ (a), IL-1 $\alpha$ (b), IL-6 (c), and IL-10 (d) protein content in lung homogenates obtained from sham \pm CLP and TxHem \pm CLP control and SNSx (6-OHDA i.p.) groups. Tissue samples were obtained $24 \mathrm{~h}$ after CLP. Means \pm SEM, $\mathrm{n}=8-10 /$ group. ${ }^{*} \mathrm{p}<0.05$ vs. time-matched CLP-, ${ }^{* *} \mathrm{p}<0.05$ vs. control/TxHem/CLP-.

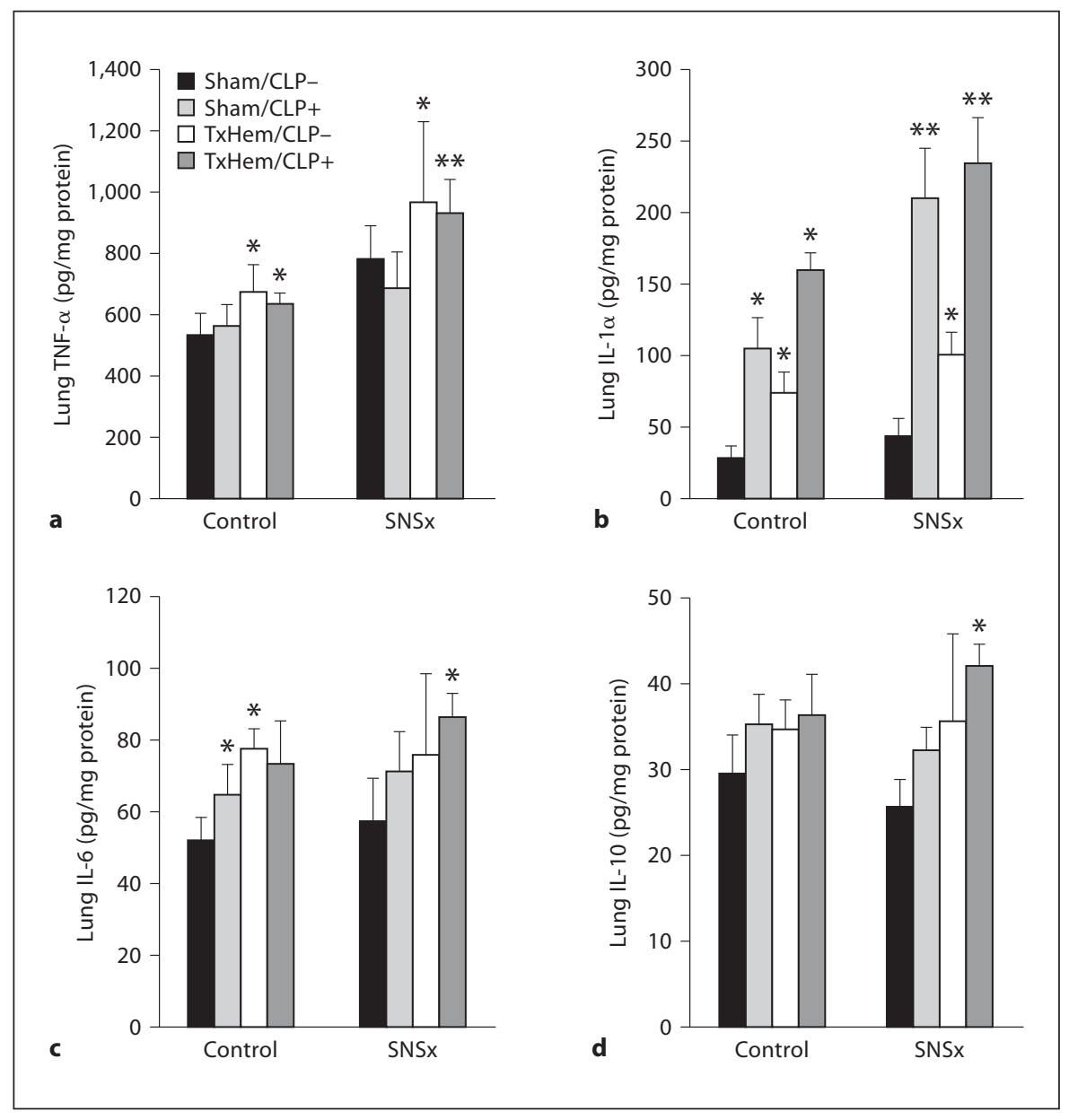

Regulation of the inflammatory response is critical to restoration of homeostasis following trauma and infection. An insufficient inflammatory response can result in immunodeficiency leading to infection [25], while an uncontrolled up-regulation of inflammatory mediators is associated with tissue injury and increased mortality [26]. While the increased mortality does not appear to be a direct result of the increased pro-inflammatory cytokine content, the consistency of IL- 6 as an early predictive marker suggests that the regulation of the inflammatory response, particularly early pro-inflammatory cytokine responses, is critical for bacterial clearance and resolution of the infectious challenge [27]. Animals in these studies were sacrificed $24 \mathrm{~h}$ after CLP, providing only a short duration for the study of survival; however, when examined in light of previous studies, the greatly enhanced IL- 6 and other pro-inflammatory cytokine levels $6 \mathrm{~h}$ after CLP suggest that SNSx animals would have likely experienced greater mortality.

Noradrenergic System Integrity and Inflammation
The current study elaborated on previous findings of dysregulation of the inflammatory response to hemorrhage following SNSx by examining the impact of tissue norepinephrine depletion on a two-hit model of hemorrhage and infection. This study utilized a model of chemical SNSx, which we have previously demonstrated to result in $80-90 \%$ depletion of tissue norepinephrine without affecting epinephrine content [14]. The depletion is further evident in the significantly impaired hemorrhage-induced rise in circulating norepinephrine levels. Circulating levels of epinephrine were unaltered by prior SNSx in this study as well as previous work. Thus, the observed effects in the present study dissect the contribution of tissue noradrenergic effects from those that are mediated by circulating levels of adrenal-derived catecholamines.

Distinct differences in the compartmental inflammatory response were noted in this study when comparing the cytokine content in plasma and peritoneal compart-

Neuroimmunomodulation 2010;17:349-358 
Fig. 6. TNF- $\alpha$ (a), IL-1 $\alpha$ (b), IL-6 (c), and IL-10 (d) protein content in spleen homogenates obtained from sham \pm CLP and hemorrhaged \pm CLP control and SNSx (6OHDA i.p.) groups. Tissues were obtained $24 \mathrm{~h}$ after CLP. Means \pm SEM, $\mathrm{n}=8-10 /$ group. ${ }^{*} \mathrm{p}<0.05$ vs. time-matched CLP-.

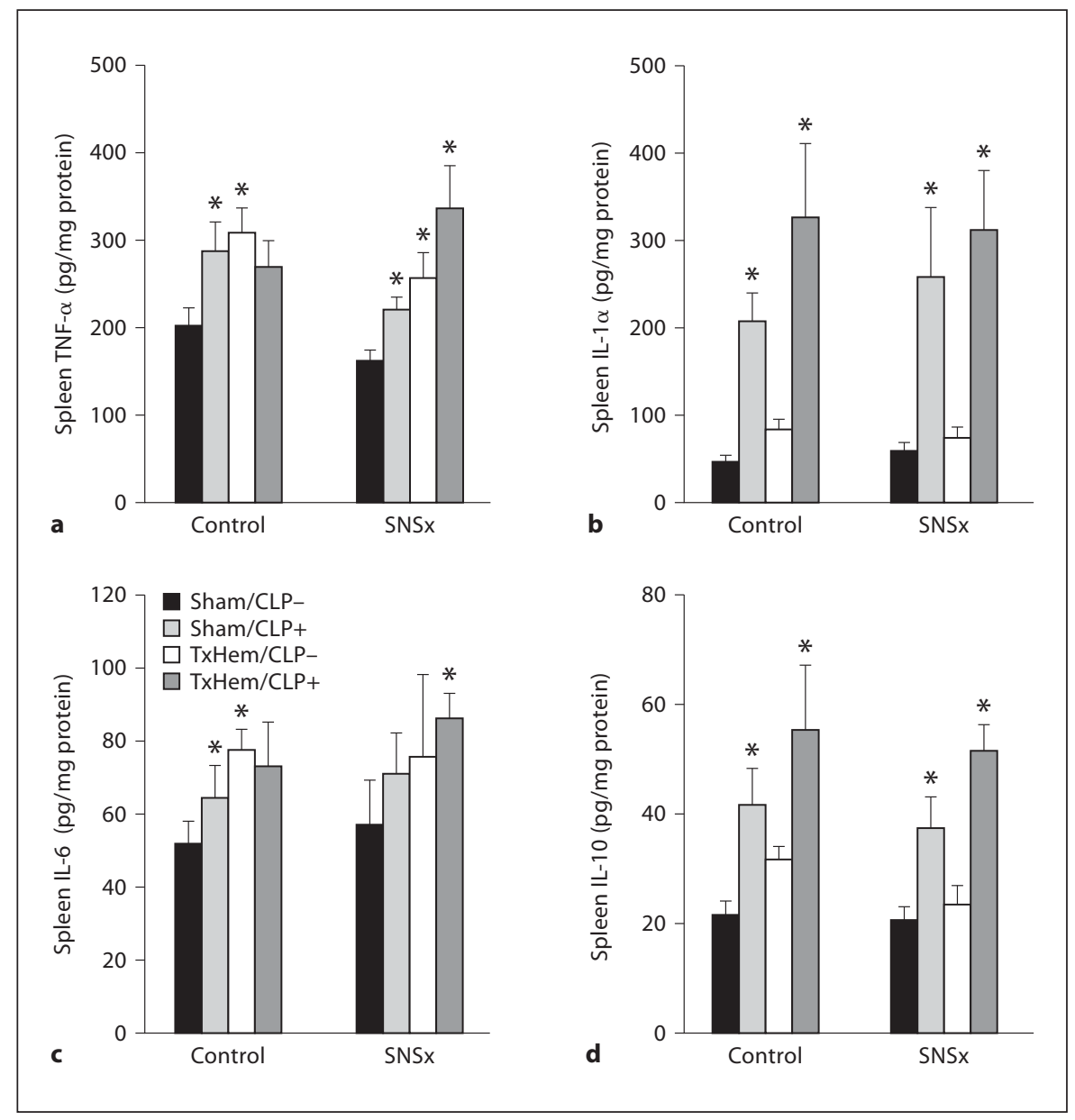

ments with the spleen content. Circulating levels of proinflammatory cytokines, as well as peritoneal lavage content, were enhanced by SNSx. In contrast, spleen cytokine content was unaltered by prior SNSx in response to CLP. A possible explanation for the discrepancy between these compartments is the differential expression of adrenergic receptor subtypes on circulating and splenic inflammatory cells. $\beta$-Adrenergic receptors are expressed on nearly all lymphoid cells [28]. $\alpha$-Adrenergic receptor expression, however, may be restricted to certain cell populations, including peritoneal macrophages and circulating monocytes, and regulation of this expression may be dependent on prevailing inflammatory cytokine levels $[29,30]$. The combination of both $\alpha$ - and $\beta$-adrenergic receptors on circulating and peritoneal lymphoid cells provides an additional potential mechanism of inflammatory response modulation by prevailing tissue levels of norepinpehrine that is largely absent in resident inflammatory cells.
In addition to dysregulation of the inflammatory response, SNSx also resulted in an accentuated and prolonged hypothermic response to CLP in hemorrhaged animals. Fever is often considered an adaptive response to infection and has traditionally been associated with increased levels of pro-inflammatory cytokines [31]. The exact role of these cytokines in the production and regulation of the hyperthermic response remains to be elucidated. Central nervous system IL-1 and IL- 6 have been demonstrated to play a role in the initial production of the fever response $[32,33]$. TNF- $\alpha$, in contrast, has been demonstrated to regulate the magnitude of the thermoregulatory response to sepsis, even potentially serving as an anti-pyretic. At physiological levels, TNF- $\alpha$ acts as an anti-pyretic, limiting sepsis or LPS-induced fever via peripheral mechanisms [32, 33]. Additionally, administration of TNF- $\alpha$-soluble receptors can prevent hypothermic responses to LPS [34]. It is possible that the SNSx-enhanced TNF- $\alpha$ response to CLP may have accentuated 
Fig. 7. TNF- $\alpha$ (a), IL-1 $\alpha$ (b), IL-6 (c), and IL-10 (d) levels in peritoneal lavage fluid obtained from sham \pm CLP and TxHem \pm CLP control and SNSx (6-OHDA i.p.) groups. Samples were obtained $24 \mathrm{~h}$ after CLP. Means \pm SEM, $\mathrm{n}=5-10$ /group. ${ }^{*} \mathrm{p}<$ 0.05 vs. time-matched CLP-, ${ }^{* *} \mathrm{p}<0.05$ vs. control/TxHem/CLP-.
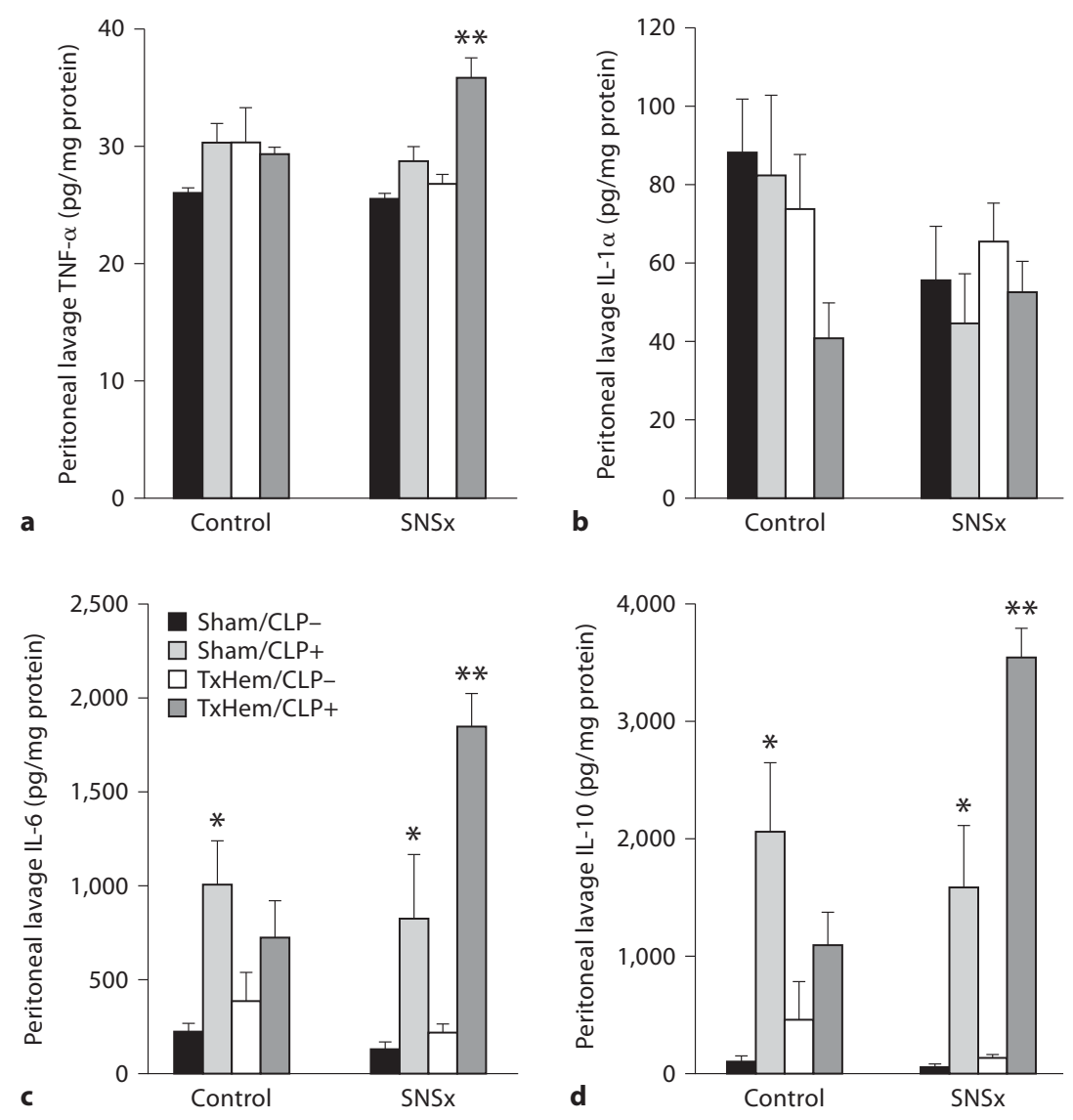

the hypothermic response to CLP and prevented any later hyperthermic response.

In conclusion, SNSx led to an impaired response to trauma and hemorrhagic shock that was associated with an accentuated inflammatory response during the early recovery period. When subjected to a 'second-hit' infectious challenge, SNSx animals demonstrated greater circulating cytokine concentrations and higher levels of pro-inflammatory cytokines in the lung and peritoneal lavage fluid. Taken together with previous findings in this model, these results suggest that the noradrenergic response, as mediated by tissue norephinephrine, contributes not only to initial hemodynamic recovery following injury and blood loss, but continues to play a significant and lasting regulatory role during the early recovery period in response to infection.

\section{Acknowledgments}

The authors would like to thank Curtis Vande Stouwe and Tesslyn Land for their excellent technical assistance. This research was supported by Office of Naval Research Grant N0001497-1-0248, Department of Defense Grant PR-054196, Louisiana Board of Regents 110350057A, and the NIAAA AA7577.

References

Neuroimmunomodulation 2010;17:349-358
Tanaka S, Matsuda Y, Shibamoto T, Wang HG: Changes in tissue blood flow and sympathetic activities to various organs during prolonged hemorrhagic hypotension in monkeys. J Auton Nerv Syst 1996;60:121128.

2 Spengler RN, Chensue SW, Giacherio DA, Blenk N, Kunkel SL: Endogenous norepinephrine regulates tumor necrosis factor-alpha production from macrophages in vitro. J Immunol 1994;152:3024-3031. 
-3 van der Poll T, Coyle SM, Barbosa K, Braxton CC, Lowry SF: Epinephrine inhibits tumor necrosis factor-alpha and potentiates interleukin 10 production during human endotoxemia. J Clin Invest 1996;97:713-719.

$\checkmark 4$ Woiciechowsky C, Asadullah K, Nestler D, et al: Sympathetic activation triggers systemic interleukin-10 release in immunodepression induced by brain injury. Nat Med 1998;4: 808-813.

5 Bulloch K, Pomerantz W: Autonomic nervous system innervation of thymic-related lymphoid tissue in wildtype and nude mice. J Comp Neurol 1984;228:57-68.

$\checkmark 6$ Nance DM, Sanders VM: Autonomic innervation and regulation of the immune system (1987-2007). Brain Behav Immun 2007;21: 736-745.

7 Spengler RN, Allen RM, Remick DG, Strieter RM, Kunkel SL: Stimulation of alpha-adrenergic receptor augments the production of macrophage-derived tumor necrosis factor. J Immunol 1990;145:1430-1434.

-8 Le Tulzo Y, Shenkar R, Kaneko D, et al: Hemorrhage increases cytokine expression in lung mononuclear cells in mice: involvement of catecholamines in nuclear factor- $\kappa \mathrm{B}$ regulation and cytokine expression. J Clin Invest 1997;99:1516-1524.

-9 Severn A, Rapson NT, Hunter CA, Liew FY: Regulation of tumor necrosis factor production by adrenaline and beta-adrenergic agonists. J Immunol 1992;148:3441-3445.

- 10 Delrue-Perollet C, Li KS, Vitiello S, Neveu PJ: Peripheral catecholamines are involved in the neuroendocrine and immune effects of LPS. Brain Behav Immun 1995;9:149162.

-11 Rice PA, Boehm GW, Moynihan JA, Bellinger DL, Stevens SY: Chemical sympathectomy increases numbers of inflammatory cells in the peritoneum early in murine listeriosis. Brain Behav Immun 2002;16:654662.

12 Pfeifer R, Tarkin IS, Rocos B, Pape HC: Patterns of mortality and causes of death in polytrauma patients - has anything changed? Injury 2009;40:907-911.
13 Keel M, Trentz O: Pathophysiology of polytrauma. Injury 2005;36:691-709.

14 Molina PE: Noradrenergic inhibition of TNF upregulation in hemorrhagic shock. Neuroimmunomodulation 2001;9:125-133.

15 Breese GR, Traylor TD: Effect of 6-hydroxydopamine on brain norepinephrine and dopamine evidence for selective degeneration of catecholamine neurons. J Pharmacol Exp Ther 1970;174:413-420.

$>16$ Molina PE, Abumrad NN: Differential effects of hemorrhage and LPS on tissue TNFalpha, IL-1 and associate neuro-hormonal and opioid alterations. Life Sci 2000;66:399409.

17 Phelan H, Stahls P, Hunt J, Bagby GJ, Molina PE: Impact of alcohol intoxication on hemodynamic, metabolic, and cytokine responses to hemorrhagic shock. J Trauma 2002;52: 675-682.

18 Hubbard WJ, Choudhry M, Schwacha MG, et al: Cecal ligation and puncture. Shock 2005;24(suppl 1):52-57.

19 Lowry OH, Rosebrough NJ, Farr AL, Randall RJ: Protein measurement with the Folin phenol reagent. J Biol Chem 1951;193:265275.

20 Elenkov IJ, Wilder RL, Chrousos GP, Vizi ES: The sympathetic nerve - an integrative interface between two supersystems: the brain and the immune system. Pharmacol Rev 2000;52:595-638.

-21 Farmer P, Pugin J: $\beta$-Adrenergic agonists exert their 'anti-inflammatory' effects in monocytic cells through the IкB/NF- $\kappa$ B pathway. Am J Physiol Lung Cell Mol Physiol 2000;279:L675-L682.

$\checkmark 22$ Ollivier V, Parry GC, Cobb R, de Prost D, Mackman N: Elevated cyclic AMP inhibits $\mathrm{NF}-\kappa \mathrm{B}$-mediated transcription in human monocytic cells and endothelial cells. J Biol Chem 1996;271:20828-20835.

$\checkmark 23$ Ye RD: $\beta$-Adrenergic agonists regulate NF$\kappa \mathrm{B}$ activation through multiple mechanisms. Am J Physiol Lung Cell Mol Physiol 2000; 279:L615-L617.

24 Graham C, Parry N, Mackman N: Role of cyclic AMP response element-binding protein in cyclic AMP inhibition of NFKB-mediated transcription. J Immunol 1997; 159:54505456.
25 Abraham E: Physiologic stress and cellular ischemia: relationship to immunosuppression and susceptibility to sepsis. Crit Care Med 1991;19:613-618.

26 Wang, H: HMG-1 as a late mediator of endotoxin lethality in mice. Science $1999 ; 285$ : 248-251.

-27 Remick DG, Bolgos GR, Siddiqui J, Shin J, Nemzek JA: Six at six: interleukin-6 measured $6 \mathrm{~h}$ after the initiation of sepsis predicts mortality over 3 days. Shock 2002;17: 463-467.

28 Kohm AP, Sanders VM: Norepinephrine and beta 2 -adrenergic receptor stimulation regulate CD4+ T and B lymphocyte function in vitro and in vivo. Pharmacol Rev 2001;53: 487-525.

29 Heijnen CJ, Rouppe van der Voort C, van de Pol M, Kavelaars A: Cytokines regulate alpha(1)-adrenergic receptor mRNA expression in human monocytic cells and endothelial cells. J Neuroimmunol 2002;125:66-72.

$>30$ Elenkov IJ, Wilder RL, Chrousos GP, Vizi ES: The sympathetic nerve - an integrative interface between two supersystems: the brain and the immune system. Pharmacol Rev 2000;52:595-638.

31 Leon LR, White AA, Kluger MJ: Role of IL-6 and TNF in thermoregulation and survival during sepsis in mice. Am J Physiol 1998;275: R269-R277.

>32 Klir JJ, McClellan JL, Kozak W, Szelényi Z, Wong GH, Kluger MJ: Systemic but not central administration of tumor necrosis factoralpha attenuates LPS-induced fever in rats. Am J Physiol 1995;268:R480-R486.

>33 Kozak W, Kluger MJ, Soszynski D, Conn CA, Rudolph K, Leon LR, Zheng H: IL- 6 and IL-1 beta in fever. Studies using cytokine-deficient (knockout) mice. Ann NY Acad Sci 1998;856:33-47.

$>34$ Long NC, Morimoto A, Nakamori T, Murakami N: Systemic injection of TNF-alpha attenuates fever due to IL-1 beta and LPS in rats. Am J Physiol 1992;263:R987-R991. 